TAIWANESE JOURNAL OF MATHEMATICS

Vol. 2, No. 4, pp. 427-434, December 1998

\title{
A WAVELET METHOD FOR THE FIRST KIND INTEGRAL EQUATIONS WITH KERNEL $k(x-y)$
}

\author{
Xiao-Qing Jin* and Jin-Yun Yuan
}

\begin{abstract}
We study the first kind integral equation

$$
\int_{0}^{+\infty} k(x-y) \sigma(y) d y=g(x)
$$

by the wavelet method. The integral equation is discretized with respect to two different wavelet bases. We then have two different linear systems. One of them is a Toeplitz system and the other one is a system with condition number $\kappa=O(1)$ after a diagonal scaling. By using the preconditioned conjugate gradient (PCG) method with the fast wavelet transform (FWT) and the fast Fourier transform (FFT), we can solve the systems in $O(n \log n)$ operations where $n$ is the size of the systems.
\end{abstract}

\section{INTRODUCTION}

In this paper, we study the first kind integral equation

$$
\int_{0}^{+\infty} k(x-y) \sigma(y) d y=g(x)
$$

by the wavelet method. The integral equation is discretized with respect to two different orthonormal wavelet bases $\mathcal{B}_{1}$ and $\mathcal{B}_{2}$. The $\mathcal{B}_{1}$ comes from the father wavelet $\varphi(x)$ and the $\mathcal{B}_{2}$ comes from the mother wavelet $\psi(x)$. After discretizing of the integral equation with respect to $\mathcal{B}_{1}$ and $\mathcal{B}_{2}$ on a finite

Received February 20, 1997.

Communicated by F.-B. Yeh.

1991 Mathematics Subject Classification: 65F35, 65N30.

Key words and phrases: Integral equation, kernel, wavelet transform, Toeplitz matrix, Sobolev space, PCG method.

${ }^{*}$ Research supported by HKRGC grant no. CUHK 316/94E and a research grant from the University of Macau. 
interval, we then have two different $n$-by- $n$ linear systems. One of them is a Toeplitz system

$$
T_{n} x=b
$$

(corresponding to $\mathcal{B}_{1}$ ), and the other one is a system $A_{n} y=d$ (corresponding to $\mathcal{B}_{2}$ ) with condition number

$$
\kappa\left(D_{n}^{-1 / 2} A_{n} D_{n}^{-1 / 2}\right)=O(1)
$$

after a diagonal scaling $D_{n}$. The relation between $T_{n}$ and $A_{n}$ is

$$
A_{n}=W_{n} T_{n} W_{n}^{-1}
$$

where $W_{n}$ is the wavelet transform matrix between $\mathcal{B}_{1}$ and $\mathcal{B}_{2}$.

We then solve the Toeplitz system $T_{n} x=b$ by solving its equivalent form

$$
A_{n} \tilde{x}=\tilde{b}
$$

with $\tilde{x}=W_{n} x$ and $\tilde{b}=W_{n} b$. For solving the system $A_{n} \tilde{x}=\tilde{b}$, we use the PCG method with the diagonal preconditioner $D_{n}$. More precisely, instead of solving the system $A_{n} \tilde{x}=\tilde{b}$, we solve the preconditioned system

$$
D_{n}^{-1} A_{n} \tilde{x}=D_{n}^{-1} \tilde{b}
$$

The condition number of the preconditioned system is, by (1),

$$
\kappa\left(D_{n}^{-1} A_{n}\right)=\kappa\left(D_{n}^{-1 / 2} A_{n} D_{n}^{-1 / 2}\right)=O(1) .
$$

When the PCG method is applied to solve the preconditioned system, the convergence rate will be linear, see [5]. By using the FWT (see [1] and [3]) and Strang's algorithm (see [2] and [7]), we can solve the system $A_{n} \tilde{x}=\tilde{b}$ and also $T_{n} x=b$ in $O(n \log n)$ operations.

The outline of the paper is as follows. In Section 2, we introduce the father wavelet basis $\mathcal{B}_{1}$ and the mother wavelet basis $\mathcal{B}_{2}$. The integral equation is discretized with respect to the bases $\mathcal{B}_{1}$ and $\mathcal{B}_{2}$. Two different linear systems are obtained. In Section 3, we use the PCG method to solve the linear systems obtained in Section 2. We then discuss the condition number and the operation cost of the PCG method.

\section{Discretization of Integral Equation}

Let $H^{s}(\mathbb{R})$ with $s \geq 0$ and $H^{t}(\mathbb{R})$ be two Sobolev spaces. The integral operator $A$ from $H^{s}(\mathbb{R})$ to $H^{t}(\mathbb{R})$ is defined as 


$$
(A \sigma)(x)=\int_{0}^{+\infty} k(x-y) \sigma(y) d y
$$

where $k(x-y) \in L^{2}(\mathbb{R})$ is symmetric, i.e., $k(x-y)=k(y-x)$. For a given function $g \in H^{t}(\mathbb{R})$, we try to find $\sigma \in H^{s}(\mathbb{R})$ such that

$$
A \sigma=g
$$

i e.,

$$
\int_{0}^{+\infty} k(x-y) \sigma(y) d y=g(x)
$$

The equivalent variational form is: find $\sigma \in H^{s}(\mathbb{R})$ such that

$$
B(\sigma, \mu)=F(\mu)
$$

for all $\mu \in H^{s}(\mathbb{R})$, where

$$
B(\sigma, \mu)=\langle A \sigma, \mu\rangle=\int_{0}^{+\infty} \int_{0}^{+\infty} k(x-y) \sigma(y) \mu(x) d y d x
$$

and

$$
F(\mu)=\int_{0}^{+\infty} g(x) \mu(x) d x
$$

We assume that $B(\sigma, \mu)$ is a continuous elliptic bilinear form on $H^{s}(\mathbb{R}) \times H^{s}(\mathbb{R})$, i.e., there exist two constants $\beta \geq \alpha>0$, such that

$$
\alpha\|\sigma\|_{H^{s}} \leq B(\sigma, \sigma) \text { and } B(\sigma, \mu) \leq \beta\|\sigma\|_{H^{s}}\|\mu\|_{H^{s}} .
$$

For instance, when $s=0$ and $k(x-y)$ is bounded with $k(x-y) \geq c>0$, then obviously, $B(\sigma, \mu)$ is a continuous elliptic bilinear form on $L^{2}(\mathbb{R}) \times L^{2}(\mathbb{R})$.

\subsection{Wavelet Bases}

Now we are going to discretize the integral equation with respect to two different orthonormal wavelet bases $\mathcal{B}_{1}$ and $\mathcal{B}_{2}$. First of all, we introduce a function $\varphi(x) \in L^{2}(\mathbb{R})$ called the father wavelet (or scaling function), with a compact support $[0, a], a>0$; see [4]. The $\varphi(x)$ has the property that

$$
\varphi(x-k), \quad k \in Z,
$$

form an orthonormal sequence in $L^{2}(\mathbb{R})$. Let $V_{0}$ be the closed linear subspace of $L^{2}(\mathbb{R})$ generated by (4). The multiresolution analysis (MRA), depending on this $\varphi(x)$, is given as follows: 
- (i) $f(x) \in V_{0}$ if and only if $f\left(2^{j} x\right) \in V_{j}$;

- (ii) $\cdots \subset V_{j-1} \subset V_{j} \subset V_{j+1} \subset \cdots$;

- (iii) $\overline{\cup_{-\infty}^{\infty} V_{j}}=L^{2}(\mathbb{R})$ and $\cap_{-\infty}^{\infty} V_{j}=0$;

- (iv) The sequence (4) forms a Riesz basis of $V_{0}$.

Let $W_{j}$ denote the orthogonal complement of $V_{j}$ in $V_{j+1}$, i.e., $V_{j+1}=V_{j} \oplus$ $W_{j}$. From MRA (iii), we also have $\oplus_{-\infty}^{\infty} W_{j}=L^{2}(\mathbb{R})$. There exists at least one function $\psi(x) \in W_{0}$ such that

$$
\psi(x-k), \quad k \in Z,
$$

is an orthonormal basis of $W_{0}$ (see [3] and [6]). The $\psi(x)$ is called the mother wavelet. We then construct the following two wavelet sequences:

$$
\varphi_{j, k}(x)=2^{j / 2} \varphi\left(2^{j} x-k\right), \quad j, k \in Z,
$$

and

$$
\psi_{j, k}(x)=2^{j / 2} \psi\left(2^{j} x-k\right), \quad j, k \in Z .
$$

The wavelet sequence $\left\{\psi_{j, k}(x)\right\}$ forms a Riesz basis of $H^{s}(\mathbb{R})$ for $s \geq 0$ (see $[6])$.

The operator $A$ defined by (2) can be projected on the subspace $V_{J}$ ( $J$ is fixed) with respect to the following two bases in $V_{J}$ :

$$
\mathcal{B}_{1}=\left(\varphi_{J, k}(x)\right)_{k}, \quad k \in Z,
$$

and

$$
\mathcal{B}_{2}=\bigcup_{-\infty<j \leq J-1}\left(\psi_{j, k}(x)\right)_{k}, \quad k \in Z
$$

Here $\mathcal{B}_{1}$ comes from the father wavelet $\varphi(x)$ and $\mathcal{B}_{2}$ comes from the mother wavelet $\psi(x)$.

\subsection{Projection of $\mathrm{A}$ with respect to $\mathcal{B}_{1}$ and $\mathcal{B}_{2}$}

Let $A_{J}$ denote the projection of $A$ on $V_{J}$. The matrix representation of $A_{J}$ corresponding to the basis $\mathcal{B}_{1}$ has the elements given by

$$
t_{p, q}=\left\langle A\left(\varphi_{J, p}\right), \varphi_{J, q}\right\rangle=\int_{0}^{+\infty} \int_{0}^{+\infty} k(x-y) \varphi_{J, p}(x) \varphi_{J, q}(y) d y d x, \quad \forall p, q .
$$

For all $\sigma, \mu \in H^{s}(\mathbb{R})$, let $\sigma_{J}, \mu_{J}$ denote the projections of $\sigma, \mu$ on $V_{J}$ respectively. Then equation (3) becomes 


$$
\int_{0}^{+\infty} \int_{0}^{+\infty} k(x-y) \sigma_{J}(y) \mu_{J}(x) d y d x=\int_{0}^{+\infty} g(x) \mu_{J}(x) d x .
$$

Let

$$
\sigma_{J}=\sum_{p} x_{p} \varphi_{J, p} \quad \text { and } \quad \mu_{J}=\varphi_{J, q}, \quad \forall q .
$$

Substituting (7) into (6), we have the following linear system

$$
T_{\infty} x=b,
$$

where $\left(T_{\infty}\right)_{p, q}=t_{p, q}$ is given by $(5)$, and

$$
(x)_{p}=x_{p}, \quad(b)_{q}=\int_{0}^{+\infty} g(x) \varphi_{J, q}(x) d x .
$$

Since $k(x-y)$ is symmetric and $\varphi(x)$ has the compact support [0,a], we have

$$
\begin{aligned}
t_{p, q} & =\int_{0}^{+\infty} \int_{0}^{+\infty} k(x-y) \varphi_{J, p}(x) \varphi_{J, q}(y) d y d x \\
& =2^{J} \int_{0}^{+\infty} \int_{0}^{+\infty} k(x-y) \varphi\left(2^{J} x-p\right) \varphi\left(2^{J} y-q\right) d y d x \\
& =2^{J} \int_{2^{-J} p}^{2^{-J}(a+p)} \int_{2^{-J} q}^{2^{-j}(a+q)} k(x-y) \varphi\left(2^{J} x-p\right) \varphi\left(2^{J} y-q\right) d y d x \\
& =2^{-J} \int_{0}^{a} \int_{0}^{a} k\left[2^{-J}(x-y+p-q)\right] \varphi(x) \varphi(y) d y d x \\
& =t_{p-q}=t_{q, p} .
\end{aligned}
$$

Hence $T_{\infty}$ is a symmetric Toeplitz matrix.

The matrix representation of $A_{J}$ corresponding to the basis $\mathcal{B}_{2}$ has the elements given by

$$
a_{p, q ; s, t}=\left\langle A\left(\psi_{p, q}\right), \psi_{s, t}\right\rangle=\int_{0}^{+\infty} \int_{0}^{+\infty} k(x-y) \psi_{p, q}(x) \psi_{s, t}(y) d y d x
$$

for $-\infty<p, s<J$ and $-\infty<q, t<\infty$. Let

$$
\sigma_{J}=\sum_{p, q} y_{p, q} \psi_{p, q} \text { and } \mu=\psi_{p, q}, \quad-\infty<p<J, \quad \forall q .
$$

Substituting (10) into (6), we have the following linear system

$$
A_{\infty} y=d
$$


where $\left(A_{\infty}\right)_{p, q ; s, t}=a_{p, q ; s, t}$ is given by $(9), y=\left(y_{p, q}\right)^{T}$ and $d=\left(d_{p, q}\right)^{T}$ are vectors with $d_{p, q}=\int_{0}^{+\infty} g(x) \psi_{p, q}(x) d x$.

\section{Condition Number and Operation Cost}

We have two different linear systems obtained in the last section. One of them is the Toeplitz system (8) (corresponding to $\mathcal{B}_{1}$ ) and the other one is the system (11) (corresponding to $\mathcal{B}_{2}$ ). Now we consider the condition number of the system (11). Following the idea in [8], we first introduce the following Lemma (see [6] and [8]).

Lemma 1. Let $f=\sum_{j, k}\left\langle f, \psi_{j, k}\right\rangle \psi_{j, k}$. Then $f \in H^{s}(\mathbb{R})$ if and only if

$$
\sum_{j, k}\left|\left\langle f, \psi_{j, k}\right\rangle\right|^{2}\left(1+4^{j s}\right)<+\infty, \quad-r<s<r
$$

where $r$ is the regularity of the MRA. Moreover, since $\left\{\psi_{j, k}\right\}$ is a Riesz basis of $H^{s}(\mathbb{R})$, we also have

$$
C_{1} \sum_{j, k}\left|\left\langle f, \psi_{j, k}\right\rangle\right|^{2}\left(1+4^{j s}\right) \leq\|f\|_{H^{s}}^{2} \leq C_{2} \sum_{j, k}\left|\left\langle f, \psi_{j, k}\right\rangle\right|^{2}\left(1+4^{j s}\right),
$$

where $C_{2} \geq C_{1}>0$ are constants.

Let $\phi \in V_{J}$ with $\phi=\sum_{j, k} w_{j, k} \psi_{j, k}$. We have

$$
\langle A(\phi), \phi\rangle=\sum_{j, k} \sum_{s, t} w_{j, k} w_{s, t}\left\langle A\left(\psi_{j, k}\right), \psi_{s, t}\right\rangle=w^{T} A_{\infty} w,
$$

where $w=\left(w_{j, k}\right)^{T}$ is a vector. By the assumption that $B(\sigma, \mu)=\langle A \sigma, \mu\rangle$ is a continuous elliptic bilinear form on the space $H^{s}(\mathbb{R}) \times H^{s}(\mathbb{R})$, we have

$$
C_{3}\|\phi\|_{H^{s}}^{2} \leq\langle A(\phi), \phi\rangle \leq C_{4}\|\phi\|_{H^{s}}^{2}
$$

where $C_{4} \geq C_{3}>0$ are constants. Combining (13) and (14), we have

$$
C_{3}\|\phi\|_{H^{s}}^{2} \leq w^{T} A_{\infty} w \leq C_{4}\|\phi\|_{H^{s}}^{2} .
$$

By using (12), one can easily obtain

$$
C_{5} \sum_{j, k}\left|2^{j s} w_{j, k}\right|^{2} \leq w^{T} A_{\infty} w \leq C_{6} \sum_{j, k}\left|2^{j s} w_{j, k}\right|^{2},
$$

where $C_{6} \geq C_{5}>0$ are constants. After a diagonal scaling $D$, we have

$$
C_{5}\|w\|^{2} \leq w^{T} D^{-1 / 2} A_{\infty} D^{-1 / 2} w \leq C_{6}\|w\|^{2},
$$


where $\|\cdot\|$ is the $l_{2}$-norm. Thus, the condition number of $A_{\infty}$ after a diagonal scaling is

$$
\kappa\left(D^{-1 / 2} A_{\infty} D^{-1 / 2}\right)=O(1) .
$$

The relation between $T_{\infty}$ given by (8) and $A_{\infty}$ given by (11) is

$$
A_{\infty}=W T_{\infty} W^{-1}
$$

where $W$ is the wavelet transform matrix between two orthonormal wavelet bases $\mathcal{B}_{1}$ and $\mathcal{B}_{2}$. We then solve the Toeplitz system (8) by solving its equivalent form

$$
\left(W T_{\infty} W^{-1}\right) W x=W b
$$

i.e.,

$$
A_{\infty} \tilde{x}=\tilde{b}
$$

where $\tilde{x}=W x$ and $\tilde{b}=W b$. For solving the system $A_{\infty} \tilde{x}=\tilde{b}$, we use the PCG method with the diagonal preconditioner $D$. Instead of solving the system $A_{\infty} \tilde{x}=\tilde{b}$, we solve the preconditioned system

$$
D^{-1} A_{\infty} \tilde{x}=D^{-1} \tilde{b}
$$

Since the condition number of the preconditioned system is

$$
\kappa\left(D^{-1} A_{\infty}\right)=\kappa\left(D^{-1 / 2} A_{\infty} D^{-1 / 2}\right)=O(1)
$$

when the PCG method is applied to solve the preconditioned system, the convergence rate will be linear (see [5]).

In practice, we usually use a finite interval instead of $[0,+\infty)$. We then have an $n$-by- $n$ system

$$
T_{n} x=b,
$$

where $T_{n}$ is the finite section of $T_{\infty}$. Let $W_{n}$ be the finite section of the wavelet transform matrix $W$. The system (15) can be solved by solving its equivalent form

$$
\left(W_{n} T_{n} W_{n}^{-1}\right) W_{n} x=W_{n} b
$$

i.e.,

$$
A_{n} \tilde{x}=\tilde{b},
$$

where $A_{n}$ is the finite section of $A_{\infty}, \tilde{x}=W_{n} x$ and $\tilde{b}=W_{n} b$. The PCG method is applied to solve the system (16) with the diagonal preconditioner $D_{n}$ which is the finite section of $D$. In each iteration of the PCG method, we have to compute the matrix-vector multiplication $A_{n} v$ for some vector $v$ and solve the 
system $D_{n} y=z$ (see [5]). For $A_{n} v$, since $A_{n} v=W_{n} T_{n} W_{n}^{-1} v$, by using the FWT, $u=W_{n}^{-1} v$ could be computed in $O(n)$ operations. The $T_{n} u$ could be computed using the FFT in $O(n \log n)$ operations by first embedding $T_{n}$ into a $2 n$-by- $2 n$ circulant matrix (see [2] and [7]). By using FWT again, we note that the operation cost for $A_{n} v$ will remain $O(n \log n)$. It requires only $O(n)$ operations to solve the system $D_{n} y=z$. Thus, the total operation cost per iteration is $O(n \log n)$. Since the number of iterations is independent of $n$, we therefore can solve the system (16) and also the system (15) in $O(n \log n)$ operations. For readers interested in the actual numerical implementation of our algorithm, we refer to [1].

\section{REFERENCES}

1. G. Beylkin, R. Coifman and V. Rokhlin, Fast wavelet transforms and numerical algorithms I, Comm. Pure Appl. Math. 44 (1991), 141-183.

2. R. Chan and G. Strang, Toeplitz equations by conjugate gradients with circulant preconditioner, SIAM J. Sci. Statist. Comput. 10 (1989), 104-119.

3. C. Chui, An Introduction to Wavelet, Academic Press, 1992.

4. I. Daubechies, Orthonormal bases of compactly supported wavelets, Comm. Pure Appl. Math. 41 (1988), 909-996.

5. G. Golub and C. Van Loan, Matrix Computation, 2nd Edition, Johns Hopkins University Press, 1989.

6. Y. Meyer, Wavelets and Operators, Cambridge University Press, 1992.

7. G. Strang, A proposal for Toeplitz matrix calculations, Stud. Appl. Math. $\mathbf{7 4}$ (1986), 171-176.

8. P. Zhang and Y. Zhang, Wavelet method for the fast resolution of natural boundary integral equation, preprint.

Xiao-Qing Jin

Faculty of Science and Technology, University of Macau

Macau

Jin-Yun Yuan

Department of Mathematics, Universidade Federal do Parana

Curitiba, Brazil 